\title{
Co-expression of keratinocyte growth factor and K-sam is an independent prognostic factor in gastric carcinoma
}

\author{
TAKAHIRO TOYOKAWA, MASAKAZU YASHIRO and KOSEI HIRAKAWA \\ Department of Surgical Oncology, Osaka City University Graduate School of Medicine, Osaka, Japan
}

Received November 3, 2008; Accepted December 19, 2008

DOI: $10.3892 /$ or_00000297

\begin{abstract}
Keratinocyte growth factor (KGF) from gastric fibroblasts have been reported to stimulate proliferation of scirrhous gastric cancer cells with K-samII amplification in a paracrine manner. The aim of this study was to evaluate the clinical significance of the co-expression of $\mathrm{K}$-sam and KGF in gastric carcinomas. A total of 136 primary gastric tumors were investigated by staining with antibodies against K-sam and KGF. K-sam expression on cancer cells and KGF expression on fibroblasts was estimated. The relationship between the K-sam and/or KGF expression and the clinicopathological characteristics were analyzed. K-sam expression was positive in 42 (31\%) of 136 gastric carcinomas. K-sam expression was positively correlated with scirrhous cancer $(\mathrm{p}<0.001)$, diffuse type $(\mathrm{p}=0.031)$, invasion depth $(p=0.018)$ and infiltration type $(p<0.001)$. Prognosis of $\mathrm{K}$-sam positive patients was significantly poorer than that of $\mathrm{K}$-sam negative patients $(\mathrm{p}<0.001)$. The prognosis of patients with both K-sam and KGF positive tumors was significantly worse in comparison to either negative tumors $(\mathrm{p}<0.001)$. In 94 patients with a curative resection, a multivariate analysis revealed the co-expression of $\mathrm{K}$-sam and $\mathrm{KGF}$ to be an independent prognostic factor $(\mathrm{p}=0.029)$. In conclusion, the co-expression of K-sam and KGF in gastric cancer might be a useful prognostic factor.
\end{abstract}

\section{Introduction}

Gastric carcinoma is one of the most frequent causes of cancer death (1). Scirrhous gastric carcinoma (diffusely infiltrating carcinoma, or linitis plastica-type carcinoma), which is characterized by cancer cell infiltration and proliferation with extensive fibrosis, especially carry a worse prognosis compared to other types of gastric carcinomas. The K-sam

Correspondence to: Dr Masakazu Yashiro, Department of Surgical Oncology, Osaka City University Graduate School of Medicine, 1-4-3 Asahi-machi, Abeno-ku, Osaka 545-8585, Japan E-mail:m9312510@med.osaka-cu.ac.jp

Key words: K-sam, keratinocyte growth factor, gastric cancer, prognosis gene was originally isolated as an amplified gene from a scirrhous gastric cancer cell line, KATO-III $(2,3)$. The K-sam gene product was later found to be identical to a keratinocyte growth factor (KGF) receptor $(4,5)$. Four types of K-sam transcript isoforms were identified: K-samI, II, III, and IV (6). The K-samII cDNA is identical to that of the KGF receptor (KGFR), which shows high-affinity binding to KGF (7-10). A recent study showed that KGF from orthotopic gastric fibroblasts stimulated the proliferation of scirrhous gastric carcinoma cells $(11,12)$ in vitro, thus suggesting that the interaction between KGF production from surrounding fibroblasts and KGFR on the cancer cells might play an important role for the progression of scirrhous gastric carcinoma. The present study analyzed the relationship between K-sam and/or KGF expression and the clinicopathological characteristics to clarify the clinical significance of the expression of $\mathrm{K}$-samII and KGF in gastric carcinoma.

\section{Materials and methods}

Clinical materials. For positive and negative control, xenografts were established by injecting $10^{7}$ OCUM-2M (13) and MKN-74 (14) cell into the flanks of female artymic BALB/c nude mice. OCUM-2M was derived from scirrhous carcinomas with $K$-sam amplification. MKN-74 was derived from welldifferentiated adenocarcinoma without $K$-sam amplification. A total of 136 patients who had undergone a resection of the primary tumor and were confirmed histologically to have sporadic advanced gastric cancer, were enrolled in this study. None of the patients had undergone preoperative radiation or/and chemotherapy. Specimens and xenografts were fixed in $10 \%$ formaldehyde solution and embedded in paraffin. Sections (4- $\mu \mathrm{m}$ thick) were cut and mounted on glass slides. The pathological diagnoses and classifications were made according to Japanese Classification of Gastric Carcinoma by the Japanese Gastric Cancer Association (15).

Antibodies and reagents. A rabbit polyclonal antibody which recognizes K-sam was purchased from Immuno-Biological Laboratories Co., Ltd. (9G-915, dilution 1:40, Gunma, Japan). A mouse monoclonal antibody which recognizes KGF was purchased from R\&D Systems, Inc. (MAB2511, dilution 1:200, Minneapolis, MN). Normal goat and rabbit serum, normal rabbit and mouse immunoglobulin $\mathrm{G}$, biotinylated goat anti-rabbit and rabbit anti-mouse immunoglobulin G, streptavidin-peroxidase reagent, and diaminobenzidine were purchased from Nichirei Corporation (Tokyo, Japan). 

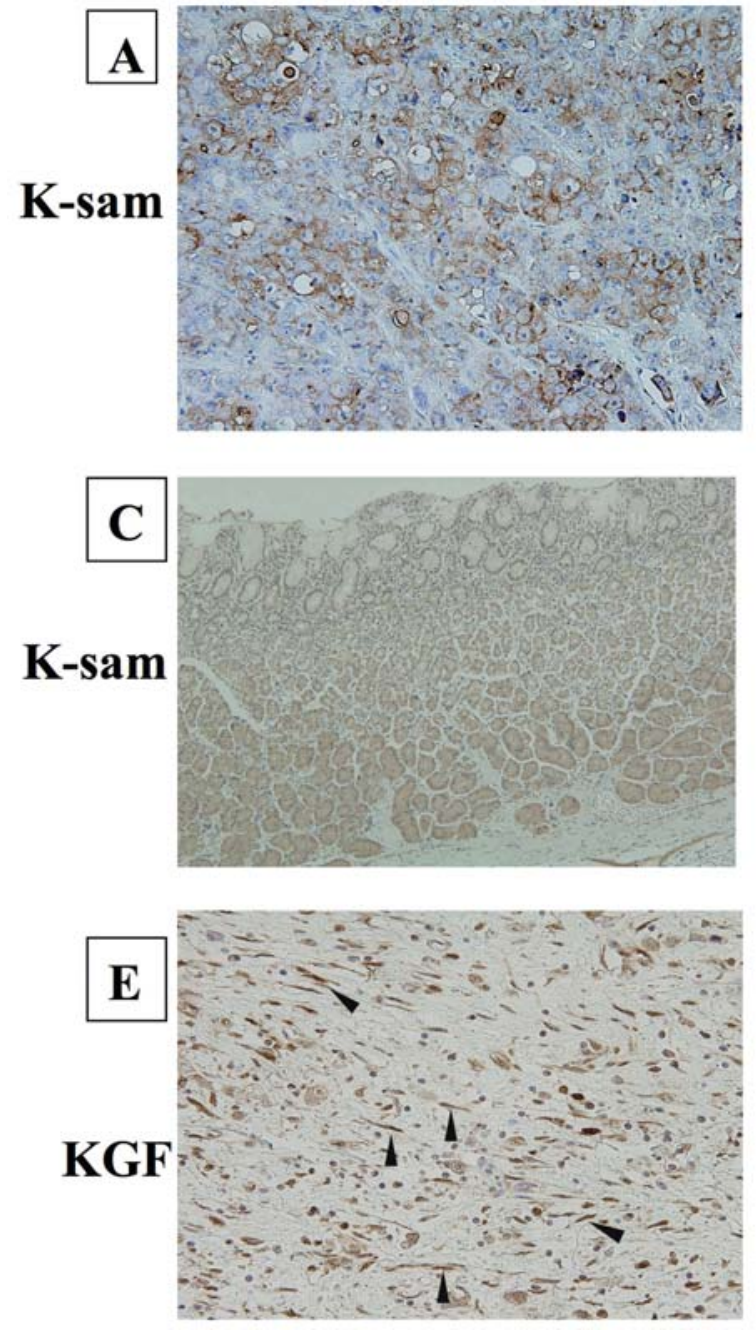

Immunohistochemical techniques. The methods for immunohistochemical determination of K-sam and KGF have been described in the manufacturer's instructions. In brief, the slides were deparaffinized in xylene and hydrated in decreasing concentrations of ethyl alcohol. The tissues were heated for $20 \mathrm{~min}$ at $105^{\circ} \mathrm{C}$ by autoclave in Target retrieval solution (Dako Co., Carpinteria, CA). Then sections were deparaffinized and incubated with $3 \%$ hydrogen peroxide in methanol for 15 min to block endogenous peroxidase activity. The sections were then washed in phosphate-buffered saline (PBS) and incubated in $10 \%$ normal goat or rabbit serum for $10 \mathrm{~min}$ to reduce non-specific antibody binding. The specimens were incubated with K-sam antibody $(2.5 \mu \mathrm{g} / \mathrm{ml})$ or KGF antibodies $(2.5 \mu \mathrm{g} / \mathrm{ml})$ overnight at $4^{\circ} \mathrm{C}$, followed by 3 washes with PBS. The sections were incubated with biotinylated goat antirabbit immunoglobulin $\mathrm{G}$ or normal mouse immunoglobulin $\mathrm{G}$ for $30 \mathrm{~min}$, followed by 3 washes with PBS. The slides were treated with streptavidin-peroxidase reagent for $15 \mathrm{~min}$ and washed with PBS 3 times. Finally, the slides were incubated in PBS diaminobenzidine and $1 \%$ hydrogen peroxide v/v for $60 \mathrm{sec}$ or $90 \mathrm{sec}$, counterstained with Mayer's hematoxylin, and mounted.

Immunohistochemical determination of $K$-sam and $K G F$. $\mathrm{K}$-sam expression was observed in the tumors by OCUM-2M cells but not in tumors by MKN-74 cells. K-sam was mainly
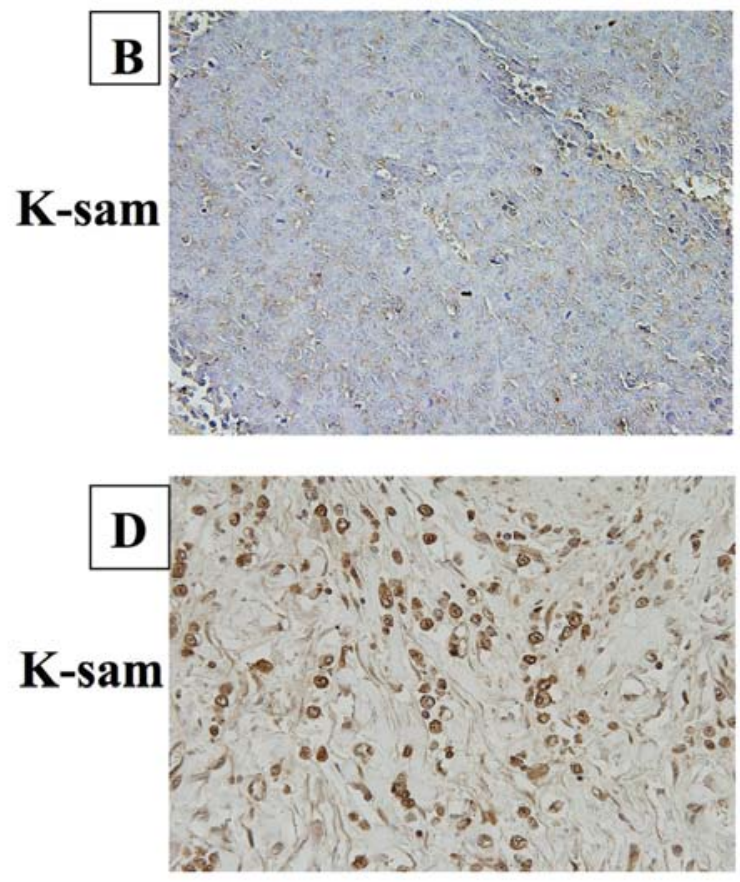

Figure 1. Immunohistochemical determination of K-sam and KGF. Expression of $\mathrm{K}$-sam was recognized at the membrane and cytoplasm of OCUM-2M cells with $K$-sam amplification (A), but not MKN-74 cells without $K$-sam amplification (B). (C) $\mathrm{K}$-sam antibody weakly stained the fundic gland in the cytoplasm in the normal stomach wall (x100). (D) Scirrhous gastric cancer cells were stained by K-sam antibody more intensely than non-cancerous cells in the cytoplasm and membrane (x400). (E) Expression of KGF was recognized at the cytoplasm of stromal cells (arrows).

immunolocalized at the membrane and cytoplasm of cancer cells (Fig. 1A). Both hematoxylin and eosin staining were used as a reference slide to select cancer areas at the invading front. The existence of cancer cells was continuously examined from the serosa to the mucosa under a microscope. The invading front was determined at the lesion where cancer cells were first found from serosal side. K-sam antibody weakly stained the gastric epithelium (Fig. 1C). The tumor cells were judged to be $\mathrm{K}$-sam positive when the tumor cells at the invading front $>25 \%$ were stained more strongly than the normal epithelium in the same sample (Fig. 1D). In addition, the fibroblasts at the invading front were judged to be KGF positive when more than two of the stromal fibroblasts were stained more intensely than the fundic gland at high magnification (x200). The slides were evaluated by two investigators (TT and MY) without any knowledge of the corresponding clinicopathological data, and when a discordance was found, the cases were then re-evaluated together and a consensus was reached.

Statistical analysis. The Chi-square test or Mann-Whitney U test was used to determine the significance of the differences between the covariates. The survival durations were calculated using the Kaplan-Meier method and analyzed by the log-rank test to compare the cumulative survival durations in the patient groups. In addition, the Cox proportional hazards model was used to compute multivariate hazards ratios for the study 


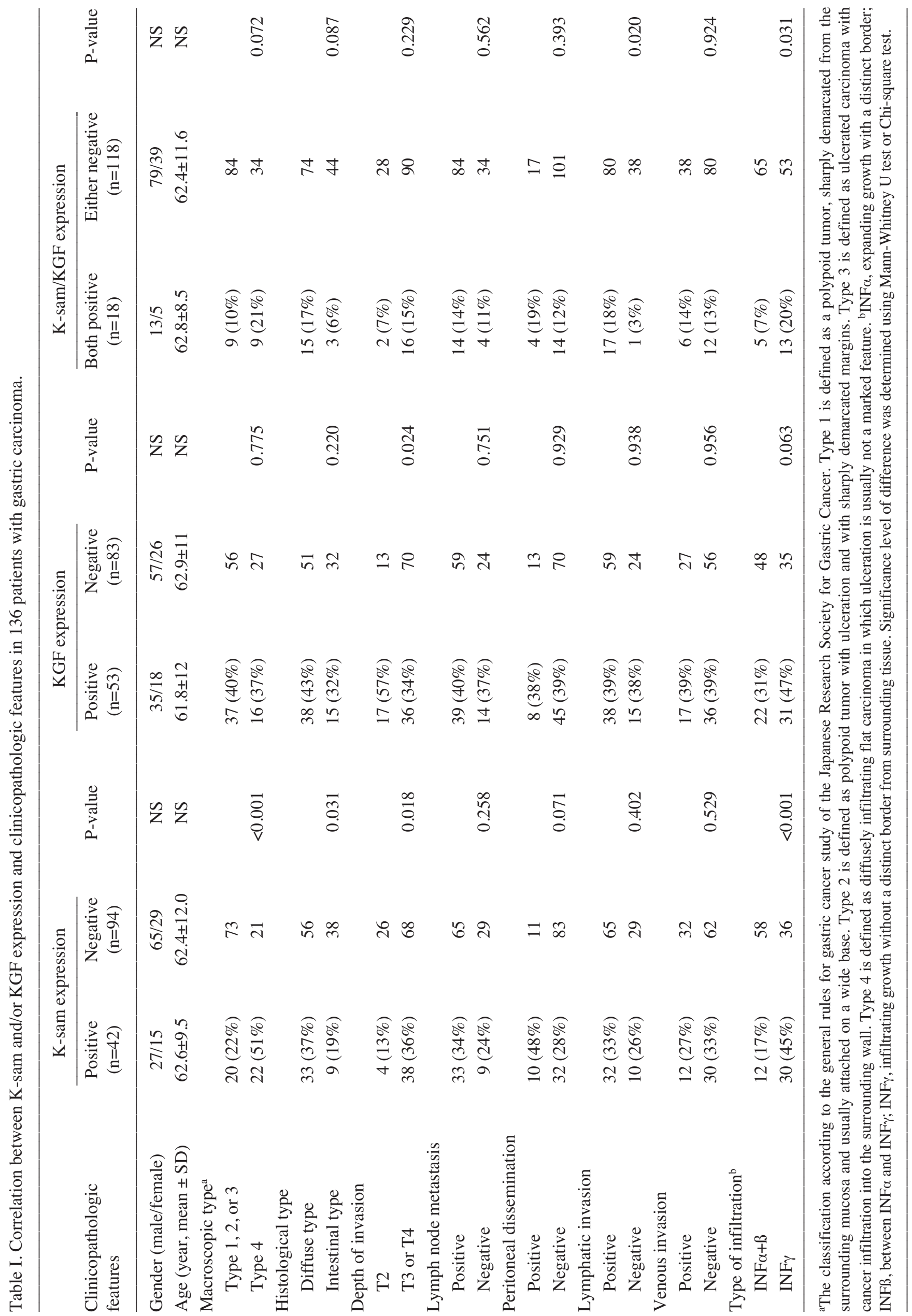


Table II. A univariate analysis with respect to the overall survival in 94 gastric cancer patients undergoing a curative resection.

\begin{tabular}{|c|c|c|c|}
\hline Parameter & Risk ratio & $95 \%$ Confidence interval & P-value \\
\hline \multicolumn{4}{|l|}{ Gender } \\
\hline Male vs. female & 2.083 & $0.965-4.497$ & 0.062 \\
\hline \multicolumn{4}{|l|}{ Co-expression of K-sam and KGF } \\
\hline Positive vs. either negative & 4.106 & $1.628-10.358$ & 0.003 \\
\hline \multicolumn{4}{|l|}{ Macroscopic type } \\
\hline Type $1+2+3$ vs. type 4 & 4.661 & $2.092-10.388$ & $<0.001$ \\
\hline \multicolumn{4}{|l|}{ Histological type } \\
\hline Diffuse type vs. intestinal type & 3.142 & $1.259-7.837$ & 0.014 \\
\hline \multicolumn{4}{|l|}{ Depth of invasion } \\
\hline T2 vs. T3 & 2.303 & $0.868-6.144$ & 0.094 \\
\hline \multicolumn{4}{|l|}{ Lymph node metastasis } \\
\hline Negative vs. positive & 1.384 & $0.602-3.184$ & 0.444 \\
\hline \multicolumn{4}{|l|}{ Lymphatic invasion } \\
\hline Negative vs. positive & 1.790 & $0.719-4.459$ & 0.211 \\
\hline \multicolumn{4}{|l|}{ Venous invasion } \\
\hline Negative vs. positive & 0.579 & $0.233-1.443$ & 0.241 \\
\hline \multicolumn{4}{|l|}{ Type of infiltration } \\
\hline INF $\alpha+\beta$ vs. INF $\gamma$ & 2.189 & $1.011-4.740$ & 0.047 \\
\hline
\end{tabular}

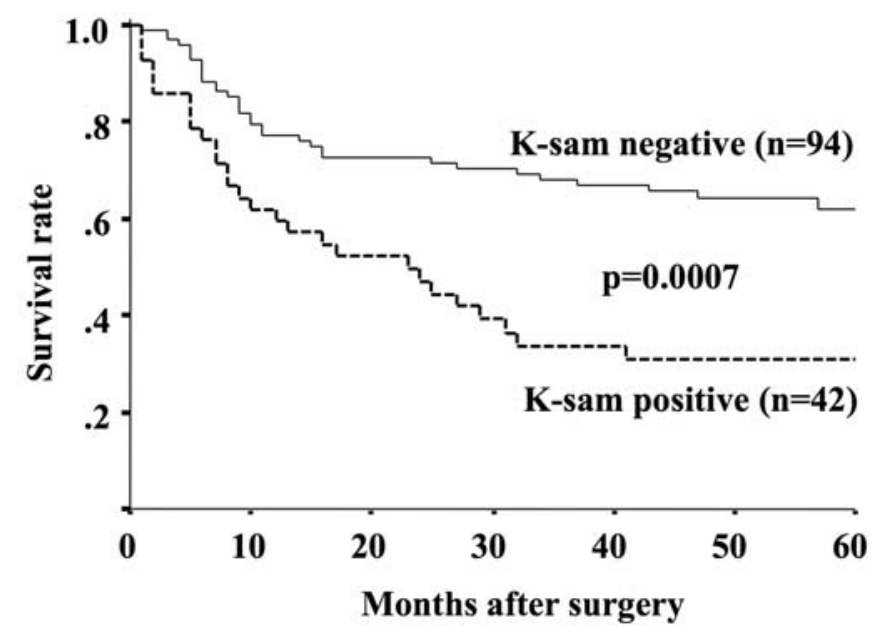

Figure 2. The overall survival of patients based on K-sam expression. The survival curve shows the Kaplan-Meier overall survival curves in relation to the K-sam expression in the gastric carcinomas. A statistically significant difference in survival was observed between the K-sam-positive and K-samnegative groups (log-rank, $\mathrm{p}=0.0007)$.

parameters. In all the tests, a $p<0.05$ was defined as statistically significant. The SPSS software program (SPSS Japan, Tokyo, Japan) was used for the analyses.

\section{Results}

Correlation between clinicopathological features and K-sam and/or KGF expression. The K-sam expression was positive in $42(31 \%)$ of 136 gastric carcinomas. The relationship between K-sam and/or KGF expression and clinicopathological features of the tumors are shown in Table I. K-sam expression was significantly present with scirrhous type $(51 \%, \mathrm{p}<0.001)$, diffuse type $(37 \%, \mathrm{p}=0.031)$, depth of invasion $(\mathrm{p}=0.018)$ and type of cancer infiltration (INF $\gamma, 45 \%$, $\mathrm{p}<0.001)$. K-sam expression tended $(\mathrm{p}<0.071)$ to be associated with peritoneal dissemination. There was no statistically significant association between K-sam expression and patient age, gender, lymph node metastasis, lymphatic invasion and venous invasion. KGF expression in stromal cells was positive in $53(39 \%)$ of 136 gastric carcinomas. KGF expression of stromal cells was significantly high at the depth of invasion (T2, 57\%) vs. T3/4, 34\%, p=0.024). The co-expression both $\mathrm{K}$-sam and KGF was positive in 18 (13\%) of 136 gastric carcinomas. K-sam and KGF co-expression was significantly correlated with lymphatic invasion $(\mathrm{p}=0.020)$ and type of infiltration (INF $\alpha+\beta, 7 \%$ vs. INF $\gamma 20 \%, \mathrm{p}=0.031)$. K-sam and KGF co-expression tended to be correlated with macroscopic type $(\mathrm{p}=0.072)$ and histological type $(\mathrm{p}=0.087)$. In contrast, there was no statistically significant association between K-sam and/or KGF and peritoneal dissemination, depth of invasion, or lymph node metastasis.

Survival. The prognosis for K-sam positive patients was significantly poorer than that of K-sam negative patients ( $\mathrm{p}=0.0007$, Fig. 2). The 5-year survival of the patients with K-sam positive tumors was $33 \%$ in comparison to $63 \%$ for those patients with negative tumors. Moreover, K-sam and $\mathrm{KGF}$ were combined, in the $\mathrm{K}$-sam positive subgroup, $\mathrm{KGF}$ 
A

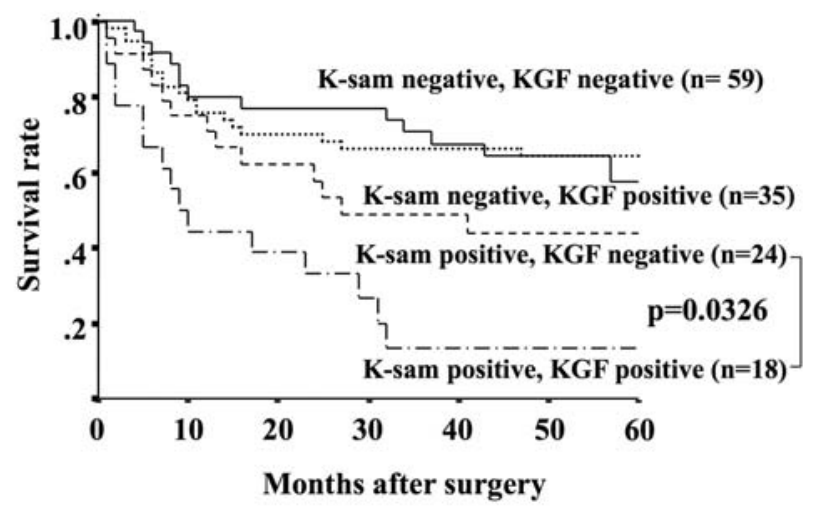

B
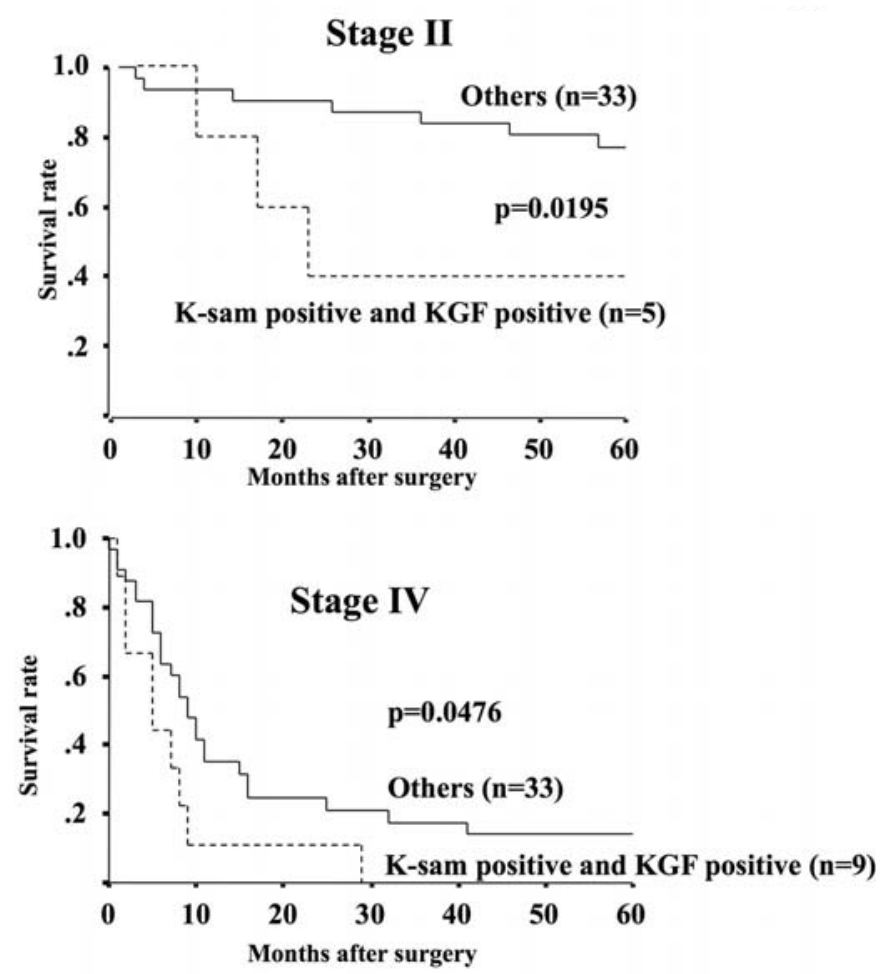

positive patients had a significantly poorer prognosis than KGF negative patients ( $\mathrm{p}=0.0326$, Fig. 3A). In stages II and IV, the prognosis of the K-sam and KGF co-expression group was significantly $(\mathrm{p}<0.05)$ poorer than that of any other groups. At stage III, the prognosis of K-sam and KGF co-expression group tended $(\mathrm{p}=0.095)$ to be poorer than those of any other groups (Fig. 3B). The prognostic markers in 94 patients of resection $\mathrm{A}$ and $\mathrm{B}$ were evaluated. In the 94 patients with a curative resection, a univariate analysis revealed the macroscopic type, histological type, type of infiltration and K-sam and KGF co-expression to all be significantly correlated with the patient survival (Table II). In a multivariate analysis, the macroscopic type and K-sam and KGF co-expression were significantly $(\mathrm{p}=0.029)$ independent prognostic factors (Table III).

\section{Discussion}

In the present study, the K-sam overexpression was significantly associated with macroscopically scirrhous type of gastric
Figure 3. The overall survivals according to the status of K-sam and/or KGF expression. (A) In patients with a positive expression of K-sam, KGF positive patients had a significantly poorer prognosis than KGF negative patients (log-rank, $\mathrm{p}=0.0326$ ). (B) In stages II and IV, the prognosis of the K-sam and KGF coexpression group was significantly $(\mathrm{P}<0.05)$ poorer than that of any other groups. In stage III, the prognosis of the K-sam and KGF coexpression group tended $(\mathrm{p}=0.095)$ to be poorer than that of any other groups.

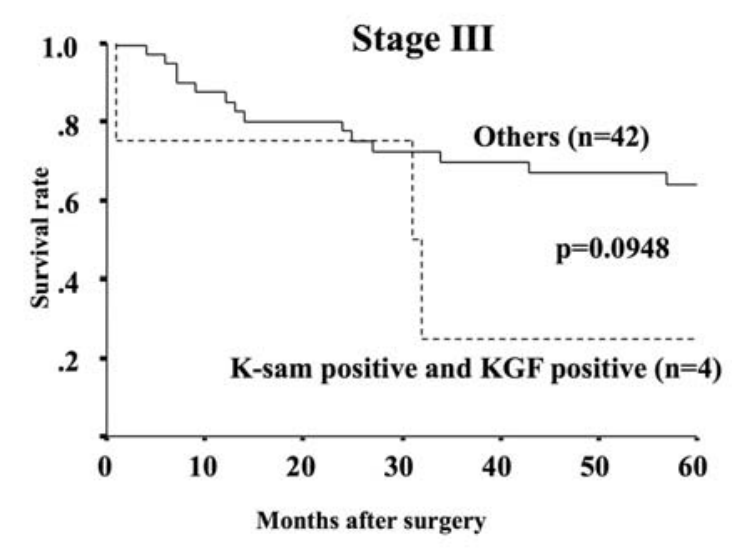

carcinoma, histologically invasive and infiltrating growth. These findings suggested that K-sam overexpression might be associated with the diffusely infiltrative and invasive growth, thus resulting in the development of the scirrhous type of gastric cancer. Previous studies demonstrated that K-sam expression is associated with distant metastasis in gastric cancer $(3,16)$ and lung cancer $(17)$. A recent study demonstrated that the peritoneal dissemination of scirrhous gastric cancer cells with $K$-sam amplification is reduced with an inhibitor of K-sam phosphorylation (18), while our data did not show any statistically significant association between the $\mathrm{K}$-sam expression and peritoneal dissemination. It might be necessary to examine whether K-sam is a useful molecule for targeting therapy for peritoneal dissemination in the future.

KGF was mainly observed expressed on fibroblasts at the cancer invasive front in this study. KGF expression of stromal cells was associated with the depth of invasion. Fibroblasts are abundantly present in either submucosa or muscularis propria lesions (19). T2 is defined as tumor invasion of muscularis propria or subserosa. T3 is defined as tumor penetration of serosa. Stromal fibroblasts have been reported to be activated partly by factors from gastric cancer cells (20). The tumor cells at the invading front play an important role for tumor-microenvironments. Therefore, KGF production might be highly expressed in the fibroblasts adjacent to the tumor cells at the invading front in $\mathrm{T} 2$, in comparison with those in T3/4. A previous study reported that KGF secreted by fibroblasts stimulated scirrhous gastric cancer cell growth in vitro $(12,13)$. These findings suggested that KGF at the cancer invasive front might enhance the proliferation and infiltrative potential of scirrhous gastric cancer cells. 
Table III. Multivariate analysis with respect to the overall survival in 94 gastric cancer patients undergoing a curative resection.

\begin{tabular}{|c|c|c|c|}
\hline Parameter & Risk ratio & $95 \%$ Confidence interval & P-value \\
\hline \multicolumn{4}{|l|}{ Co-expression of K-sam and $\mathrm{KGF}$} \\
\hline Positive vs. either negative & 2.949 & $1.114-7.805$ & 0.029 \\
\hline \multicolumn{4}{|l|}{ Macroscopic type } \\
\hline Type $1+2+3$ vs. type 4 & 3.056 & $1.172-7.968$ & 0.022 \\
\hline \multicolumn{4}{|l|}{ Histological type } \\
\hline Diffuse type vs. intestinal type & 2.014 & $0.691-5.873$ & 0.200 \\
\hline \multicolumn{4}{|l|}{ Type of infiltration } \\
\hline INF $\alpha+\beta$ vs. INF $\gamma$ & 0.918 & $0.351-2.400$ & 0.861 \\
\hline
\end{tabular}

The co-expression of KGF and K-sam was associated with lymphatic invasion and infiltrative growth. In patients with $\mathrm{K}$-sam positive, KGF expression revealed poorer prognosis compared to KGF-negative. Although there were no statistical differences in the survival between the K-sam and KGF co-expression group and any other groups in stage III, a significant difference was observed among the patients in stages II and IV. A small number $(n=4)$ of patients with K-sam and KGF co-expression might thus be one of the reasons that no significance was observed in stage III. Regarding overall survival, a multivariate analysis showed that co-expression of $\mathrm{K}$-sam and KGF were independent prognostic factors for the patients with a curative resection. These findings suggested that KGF from fibroblasts plays an important role for the progression of K-sam-positive cancer cells. This appears to be the first study to demonstrate that K-sam and KGF coexpression is a prognostic indicator in patients with gastric carcinoma.

In conclusion, the co-expression of $\mathrm{K}$-sam and $\mathrm{KGF}$ in gastric cancer is considered to be associated with the invasive and infiltrating growth, while it is also an independent prognostic factor.

\section{Acknowledgements}

This study was supported in part by a Grant-in-Aid for Scientific Research 18591475 and 18390369 from the Ministry of Education, Science, Sports, Culture and Technology of Japan, by a JSGE Grant-in-Aid for Scientific Research, by a Grant-in-Aid for Kobayashi Foundation for Innovative Cancer Chemotherapy, and by a Grant-in-Aid for the Osaka Medical Research Foundation for Incurable Diseases.

\section{References}

1. Roder DM: The epidemiology of gastric cancer. Gastric Cancer 5 (Suppl 1): 5-11, 2002.

2. Nakatani H, Tahara E, Yoshida T, et al: Detection of amplified DNA sequences in gastric cancers by a DNA renaturation method in gel. Jpn J Cancer Res 77: 849-853, 1986.

3. Nakatani H, Sakamoto H, Yoshida T, et al: Isolation of an amplified DNA sequence in stomach cancer. Jpn J Cancer Res 81: 707-710, 1990

4. Dionne CA, Crumley G, Bellot F, et al: Cloning and expression of two distinct high-affinity receptors cross-reacting with acidic and basic fibroblast growth factors. EMBO J 9: 2685-2692, 1990.
5. Hattori $\mathrm{Y}$, Odagiri $\mathrm{H}$, Nakatani $\mathrm{H}$, et al: $\mathrm{K}$-sam, an amplified gene in stomach cancer, is a member of the heparin-binding growth factor receptor genes. Proc Natl Acad Sci USA 87: 5983-5987, 1990.

6. Katoh M, Hattori Y, Sasaki H, et al: K-sam gene encodes secreted as well as transmembrane receptor tyrosine kinase. Proc Natl Acad Sci USA 89: 2960-2964, 1992.

7. Johnson DE, Lu J, Chen H, Werner S and Williams LT: The human fibroblast growth factor receptor genes: a common structural arrangement underlies the mechanisms for generating receptor forms that differ in their third immunoglobulin domain. Mol Cell Biol 11: 4627-4634, 1991.

8. Champion-Arnaud P, Ronsin C, Gilbert E, Gesnel MC, Houssaint E and Breathnach R: Multiple mRNAs code for proteins related to the BEK fibroblast growth factor receptor. Oncogene 6: 979-987, 1991.

9. Miki T, Bottaro DP, Fleming TP, et al: Determination of ligandbinding specificity by alternative splicing: two distinct growth factor receptors encoded by a single gene. Proc Natl Acad Sci USA 89: 246-250, 1992.

10. Ishii $\mathrm{H}$, Hattori $\mathrm{Y}$, Itoh $\mathrm{H}$, et al: Preferential expression of the third immunoglobulin-like domain of $\mathrm{K}$-sam product provides keratinocyte growth factor-dependent growth in carcinoma cell lines. Cancer Res 54: 518-522,1994.

11. Yashiro M, Chung YS and Sowa M: Role of orthotopic fibroblasts in the development of scirrhous gastric carcinoma. Jpn J Cancer Res 85: 883-886, 1994.

12. Nakazawa K, Yashiro M and Hirakawa K: Keratinocyte growth factor produced by gastric fibroblasts specifically stimulates proliferation of cancer cells from scirrhous gastric carcinoma. Cancer Res 63: 8848-8852, 2003.

13. Yashiro M, Chung YS, Kubo T, Hato F and Sowa M: Differential responses of scirrhous and well-differentiated gastric cancer cells to orthotopic fibroblasts. Br J Cancer 74: 1096-1103, 1996.

14. Niigata HHJ: Establishment of cultured cell lines of human stomach cancer origin and their morphological characteristics. Exp Med 91: 737-763, 1977.

15. Japanese Gastric Cancer A: Japanese Classification of Gastric Carcinoma - 2nd English edition. Gastric Cancer 1: 10-24, 1998.

16. Hattori $\mathrm{Y}$, Itoh $\mathrm{H}$, Uchino $\mathrm{S}$, et al: Immunohistochemical detection of K-sam protein in stomach cancer. Clin Cancer Res 2: 1373-1381, 1996.

17. Yamayoshi T, Nagayasu T, Matsumoto K, Abo T, Hishikawa Y and Koji T: Expression of keratinocyte growth factor/fibroblast growth factor-7 and its receptor in human lung cancer: correlation with tumour proliferative activity and patient prognosis. J Pathol 204: 110-118, 2004.

18. Nakamura K, Yashiro M, Matsuoka T, et al: A novel molecular targeting compound as K-samII/FGF-R2 phosphorylation inhibitor, Ki23057, for scirrhous gastric cancer. Gastroenterology 131: 1530-1541, 2006.

19. Guo L, Kuroda N, Nakayama H, et al: Cytokeratin-positive subserosal myofibroblasts in gastroduodenal ulcer; another type of myofibroblasts. Histol Histopathol 21: 697-704, 2006.

20. Yashiro M, Chung YS, Nishimura S, Inoue T and Sowa M: Fibrosis in the peritoneum induced by scirrhous gastric cancer cells may act as 'soil' for peritoneal dissemination. Cancer 77: 1668-1675, 1996. 